
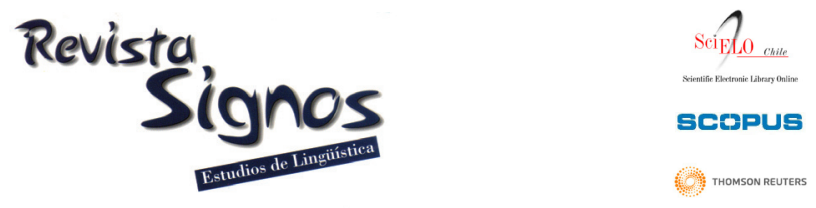

\title{
Reformulación y uso del pronombre demostrativo neutro 'esto' en la elaboración de síntesis escritas por parte de estudiantes universitarios
}

\section{Reformulation and use of the neutral demonstrative pronoun 'this' in the production of written synthesis by university students}

\section{Carmen Torres}

Universidad DE LA República

URUGUAY

carmet@adinet.com.uy

\section{Graciela Boces}

UNIVERSIDAD DE LA REPÚBLICA

URUGUAY

gboces@gmail.com

Recibido: 25-III-2011 / Aceptado: 19-XII-2011

\section{Resumen}

En este trabajo se estudia el uso del pronombre demostrativo neutro 'esto' en textos producidos por estudiantes de psicología realizados durante una tarea curricular. Como parte de los objetivos, se describen y analizan las ocurrencias del pronombre demostrativo neutro (PDN), se compara el texto fuente con el texto producido y se interpretan las operaciones psicolingüísticas que incluyen su uso en este corpus. Como resultado del análisis se proponen categorías que permitan reflejar el rol funcional que adopta el PDN en tipos de textos similares o en segmentos reformulativos de otros textos. Para su estudio se considera la dependencia composicional del género de texto y de la tarea en la que se enmarca su producción. En los textos producidos la ocurrencia del PDN alcanza una proporción muy alta en comparación con las formas pronominales no neutras. Por otra parte, se consigna en más de un $80 \%$ en el contexto de reformulación del texto fuente (TF) y en directa correspondencia con él. Se destaca la importancia de estudiar textos escritos en situaciones 'reales' en el marco de modelos relacionales. Considerando posibles dificultades de los estudiantes en la escritura, estos hallazgos ofrecen caminos promisorios para pensar aplicaciones educativas.

Palabras Clave: Estudiantes universitarios, texto, pronombre demostrativo, anáfora, reformulación. 


\begin{abstract}
In this paper it is studied the use of the neutral demonstrative pronoun 'this' in texts produced by psychology students during a curricular task. As part of the objectives, the occurrences of the neutral demonstrative pronoun (NDP) are described and analyzed, the source text and the produced text are compared and the psycholinguistic operations that include its use in this corpus are interpreted. As a result of the analysis, the categories proposed allow to show the functional role that the NDP adopts in similar types of texts or in reformulative segments of other texts. It is considered for its study the generic compositional dependence of the type of text and of the task in which it is produced. In the students' texts, the NDP reaches a high proportion in comparison with the other no-neutral pronouns. Beside, the occurrence is found in more of the $80 \%$ of the text in the context of the reformulation of the source text and in direct correspondence with it. The findings presented here offer promising paths for educational applications, considering the difficulties of students in their writings.
\end{abstract}

Key Words: Text, university students, reformulation, demonstrative pronoun, anaphora.

\title{
INTRODUCCIÓN
}

En este artículo nos proponemos estudiar el uso del demostrativo neutro 'esto' a partir de síntesis escritas por estudiantes universitarios que son producidas en el contexto de una tarea curricular. El interés de la fórmula demostrativa pronominal neutra seleccionada como objeto de estudio parece múltiple, empezando por considerar que dentro del grupo de las formas pronominales neutras, el 'esto', en español, resulta la fórmula a la que se recurre más habitualmente en la escritura. Por otra parte, este pronombre adopta funciones anafóricas que han merecido atención por su carácter vago e indeterminado. Nos interesó por esto estudiar cómo se usaba en tipos de textos muy habituales en el contexto educativo, por las características asociadas al uso de la unidad en sí y por su posible rol sintomático de las relaciones que los estudiantes entablan con los textos a nivel de su lectura y producción.

Este trabajo se enmarca en el estudio del desarrollo de géneros, operaciones y estrategias que subyacen a la escritura en entornos educativos. Entendemos que reviste gran interés estudiar los escritos de estudiantes para otorgar una dimensión práctica a la conceptualización de mecanismos y estrategias empleados en la producción y comprensión de textos, diferenciando este contexto del contexto de producción de géneros discursivos académicos especializados.

Nos proponemos describir y explicar algunos de los usos del pronombre demostrativo en los textos de los estudiantes, tomando en cuenta el supuesto de la 
dependencia del género compositivo del texto, de la tarea y las prácticas a través de tres ejes: el valor anafórico del demostrativo, el carácter reformulativo del texto producido, y las estrategias y operaciones psicolingüísticas de lectura y composición del texto. No se encuentran referencias en español sobre este punto y un interés adicional que perseguimos en la investigación fue la de modelizar una serie de relaciones que hacen posible interpretar la unidad sin las restricciones más limitativas de los enfoques gramaticales o psicolingüísticos clásicos.

Consideramos que el contexto discursivo de reformulación de otro texto puede dejar al descubierto aspectos fundamentales de la relación de los estudiantes con los textos, al permitir mostrar determinadas conexiones con el conjunto de los procesos y operaciones vinculados a la comprensión y producción de textos en contextos académicos o educativos. En este trabajo no nos ocupamos del estudio de la comprensión en particular, sin embargo, integramos el problema de la comprensión a la interpretación de los resultados, ya que partimos del supuesto de que el texto producido deriva de la interacción con el texto fuente (TF), como texto leído. Por lo que su producción requiere y/o manifiesta aspectos de los procesos de comprensión situada del texto y su contenido.

Para el tratamiento de la unidad lingüística seleccionada adoptamos un enfoque psicosociolingüístico interesado en profundizar en las relaciones entre uso, géneros discursivos y contexto de tarea y prácticas. Para ello consideramos tres niveles de interpretación, de los cuales priorizamos en este artículo los dos últimos. Estos niveles son relativos a:

- la interpretación de las relaciones entre textos, contexto de prácticas y tarea;

- el nivel lingüístico-textual y discursivo;

- las operaciones y estrategias piscolingüísticas.

Como contexto de referencia más general seguimos la influencia de los autores que promueven la consideración de hipótesis socio culturales e históricas (Bakhtin, 1985; Vygotsky, 1993; Wertsch, 1993, 1998) y socio discursivas (Schneuwly, 1994; Bronckart, 2003, 2004, 2006). Con referencia a este marco el concepto de género textual y discursivo puede interpretarse como una bisagra entre procesos sociales y psicológicos a través de la categoría de mediación, por cuanto es parte de las formas organizadas que adquiere el lenguaje comunicativo en un determinado contexto. El género discursivo, a su vez, deriva en una dialéctica que es formativa a la vez que mediadora de procesos psicológicos y aprendizajes específicos. La tarea y su contexto proponen restricciones, orientaciones y principios funcionales que interactúan con la forma y el contenido del texto. El uso, en este caso, de una unidad como el PDN, aunque se presente caracterizando el nivel lingüístico-textual y discursivo, y por lo tanto, requiera de instrumentos y categorías específicas a dicho nivel, puede interpretarse alineando los tres niveles referidos, sin que ninguno se pueda reducir a los otros, ni requiera carecer de principios funcionales específicos. 
Para la organización del artículo, a lo largo de la primera parte realizamos una revisión de la literatura sobre las características de los demostrativos, determinantes y pronominales. Nos detenemos en el PDN ubicándolo en el grupo de las expresiones deícticas o anafóricas. Caracterizamos el género de texto producido, así como del diseño y la situación de realización de la tarea. Combinamos aportes vinculados a los procesos composicionales y a la descripción lingüístico-gramatical y funcional.

La segunda parte corresponde al análisis del corpus. Nos proponemos aquí: identificar y comparar las ocurrencias de los pronombres y determinantes demostrativos, clasificar el contexto y uso del PDN, e interpretar las operaciones de reformulación del TF que incluyen su uso. A partir del análisis proponemos categorías que permitan reflejar las funciones que adopta el PDN en tipos de textos similares o en segmentos reformulativos de otros textos.

En las conclusiones, se sugiere que las condiciones de producción y los géneros de textos que forman parte de la tarea son claves para favorecer un uso de esta unidad que resulta más controlado por la búsqueda de correspondencias superficiales con el TF, antes que por una elaboración basada en la comprensión conceptual del texto de la fuente usado como guía, lo cual plantea diversas interrogantes y consecuencias.

\section{Marco referencial}

\subsection{El pronombre demostrativo neutro 'esto' en el conjunto de los demostrativos}

El grupo de los demostrativos abarca los determinantes, pronombres y adverbios. Las formas determinantes se acompañan de una categoría nominal: 'este libro', 'esta casa', admitiendo marca de género y número. En español, el 'esto' corresponde a una unidad demostrativa exclusivamente pronominal que, por ser neutra, no resulta marcada morfológicamente por la categoría de género y número (Eguren, 1999).

Según el Manual de la Real Academia Española (RAE, 2010), al grupo de los demostrativos neutros en español corresponden: 'esto', 'eso', 'aquello', 'tanto' y 'tal'. Sin embargo, los tres primeros tienen un comportamiento compartido y diferenciado del resto. Dentro de las formas del demostrativo morfológicamente próximos al 'esto', adquieren valor pronominal: 'esta', 'este' y sus plurales. El pronombre 'esto' se distingue por sus características y por la frecuencia de su uso.

El demostrativo neutro corresponde a un pronombre independiente y, como tal, representa una fórmula económica, sin significado propio definido. De Beaugrande y Dressler (2005) lo han calificado dentro del grupo de las proformas que tienden a una función básicamente sustitutiva. Aunque principalmente suele mencionarse el rol anafórico del pronombre neutro, algunos autores agregan un rol de 'enlace' de carácter semántico-pragmático entre los segmentos conectados, ubicándolo a mitad de camino 
entre anáfora y la deixis. Asimismo, se han señalado funciones argumentativas o resuntivas del PDN (Moskovit, 1983; Steinberg, Kaufer \& Geisler, 1984; Geisler, Kaufer \& Steinberg, 1985; Diessel, 1999), que se conectan con su valor anafórico. Como parte de su funcionamiento, algunos autores distinguen un uso de este pronombre demostrativo puramente demarcativo. Stirling (2001) se interesa por mostrar que esta fórmula anafórica puede funcionar como un indicador de las fronteras de la estructura del discurso. En este caso, el pronombre demostrativo simplemente indicaría fronteras textuales que podrían ser identificadas por convenciones tipográficas o locuciones relativamente libres de contenido.

Desde el punto de vista sintáctico, el 'esto' anafórico o deíctico puede contar como antecedentes con frases nominales simples, así como con frases complejas, incluidas las oraciones y los párrafos. Los contextos gramaticales en los que puede aparecer el 'esto' son variados y estarían ligados a las funciones que cumple. Puede proponerse como núcleo pronominal o resultar asociado a distintos modificadores gramaticales: ser incluido en frases preposicionales, ser antecedido por cuantificadores, así como ser seguido por relativas restrictivas o por adverbios en función de intensificadores o especificadores, etc.

\subsection{Deixis, anáfora y demostrativo neutro}

Son varios los autores que se han ocupado de la relación entre anáforas y demostrativos recurriendo a modelos gramaticales o de procesamiento psicolingüístico. Los trabajos de O’Brien, Duffy y Myers (1986), Yuill y Oakhill (1988), Cornish (1995, 2003), Diessel (1999) y Poesio, Sturt, Arstein y Filik (2006), presentan resultados sobre la investigación de procesos y mecanismos psicolingüísticos y discursivos que corresponden a la anáfora dentro de la que pueden ubicarse las funciones del pronombre demostrativo de modo más específico.

El pronombre demostrativo 'esto' ha sido especialmente tratado dentro del paradigma de unidades discursivas que adquieren función anafórica (Moskovit, 1983; Steinberg et al., 1984; Geisler, et al., 1985). Esto es, aquellas que pueden sustituir o referir a un segmento textual precedente. Las expresiones anafóricas suelen caracterizarse por su dependencia sintáctica y semántica de segmentos discursivos antecedentes. En general, se identifican como valores anafóricos no solo los que establecen una correspondencia estricta, sino también usos más laxos.

Las expresiones anafóricas han sido estudiadas desde distintos enfoques y orientaciones. La investigación gramatical, pragmática, psicolingüística y discursiva puso de manifiesto la complejidad de los mecanismos que subyacen al uso de las diferentes clases de referencia anafórica.

Las distintas formas de anáfora constituirían enlaces intratextuales que cohesionan el texto colaborando en su unidad semántica y sintáctica (Halliday \& Hasan, 1976; 
Givón, 2001). Los enfoques funcionales han destacado el servicio que presta la anáfora a la economía lingüística y a la posibilidad de progresión textual y seguimiento de la referencia. El valor anafórico se asocia a la noción de indexicalidad, compartida también con la deixis (Eguren, 1999; Stirling, 2001). Cabe considerar que la deixis y la anáfora son términos que aparecen diversamente tratados en la bibliografía. Algunos autores destacan la diferenciación específica de las formas deícticas y las demostrativas de otras categorías gramaticales (Diessel, 1999). Las perspectivas que presentan diferencias relevantes entre ambas nociones conciben que la deixis establece relaciones con el contexto extraverbal, en tanto la anáfora refiere al contexto textualdiscursivo. Algunos autores prefieren, sin embargo, destacar la relación íntima entre ambas, sobre todo en el caso de los demostrativos (Cornish, 1995; Diessel, 1999), reconociendo en la deixis un posible origen de sus usos intratextuales (Lyons, 1977).

Lyons (1977), por su parte, se refiere a 'deixis textual' para señalar los casos en los que no hay correferencialidad. Distingue entre deixis pura e impura, caracterizando esta última como aquella que no solo refiere a un segmento textual, sino que alude a otros aspectos discursivos (terceras entidades), tales como las proposiciones y la fuerza ilocutiva, por lo que podría considerarse equivalente a la deixis discursiva de algunos autores (Diessel, 1999). La RAE (2010), por su parte, da por sentado que los demostrativos son representantes característicos de las categorías deícticas, vinculadas al contexto situacional o lingüístico.

Resulta un aporte de interés el que realiza la psicolingüística evolutiva al mostrar en distintos estudios sobre adquisición del lenguaje que el manejo apropiado de las distintas formas de anáfora es tardío, tanto en el discurso oral como en el escrito (Karmiloff-Smith, 1981; Wexler \& Chien, 1985). El uso de la referencia anafórica podría mostrar una influencia selectiva y diferencial de los entornos sociales de uso del lenguaje con respecto a los usos exofóricos, como lo sugirieron estudios inspirados en Bernstein (1971).

En el texto, el PDN puede caracterizarse como una expresión anafórica de carácter intratextual. El uso de este pronombre ha sido caracterizado como opaco, puesto que su presencia no garantiza o facilita la correcta identificación del referente. Halliday y Hasan (1976) y Moskovit (1983) incluyen el demostrativo neutro en el conjunto de las fórmulas caracterizadas por contar con una 'referencia amplia' (broad reference). A diferencia de los otros pronombres, el PDN admite fácilmente un núcleo referencial que excede al nombre e, inclusive, a la oración, por lo que el antecedente se vuelve más amplio, inespecífico y ambiguo. En la producción escrita, esta ambigüedad se manifiesta especialmente cuando este demostrativo pronominal no refiere un nombre o frase nominal definido. Es así que el PDN se vuelve una fórmula sumamente económica aunque, por esto mismo, difícil de manejar. Cabe agregar que, debido a estas consideraciones, resulta insuficiente estudiarlo en los términos de una simple anáfora sin adoptar una perspectiva discursiva. 
La investigación psicolingüística y funcional se ocupó de las condiciones de accesibilidad de la referencia anafórica como un parámetro para evaluar la eficacia en la identificación de la referencia por parte del lector y la comprensión del texto (Yuill \& Oakhill, 1988; Ehrlich, 1996; Poesio et al., 2006). Este aspecto podría conectarse con las características de uso del 'esto' que venimos desarrollando.

En particular, se ha considerado que este pronombre demostrativo puede afectar la comprensión del texto, por lo que algunos enfoques prescriptivos de estilo han sugerido que se evite su uso, sobre todo en el caso de los escritores más inexperientes. En la lectura, la ambigüedad de la referencia anafórica favorece la pérdida de la continuidad del sentido del texto (Moskovit, 1983; Finn, 1995). Distintos autores han señalado la contribución que realiza a este fenómeno la falta de algún tipo de especificación tipográfica, formal o semántica, de modo tal que se haga posible convertir el antecedente en una fórmula-objeto (Moskovit, 1983; Geisler et al., 1985).

\subsection{Tipo de texto y reformulación}

El tipo de corpus que seleccionamos corresponde a escritos elaborados ante el pedido de producción de una síntesis en el contexto de una tarea de evaluación curricular. El estudio de los rasgos genéricos de las producciones escritas, así obtenidas, ya había revelado que los estudiantes suelen elaborar la síntesis según el modelo compositivo del resumen en cuanto a lo que podríamos catalogar como sus rasgos prototípicos (Torres, 2004, 2009a). Dentro de estos rasgos, consignamos como parte de la estrategia compositiva el predominio del carácter reformulativo de otro texto, en base a la abreviación y conservación del contenido informativo como principios funcionales predominantes.

Los resúmenes han sido caracterizados extensamente dentro de los géneros escolares (Bernié, 1994, 2001; Dolz \& Schneuwly, 1997). Cabe acotar, que el texto que obtuvimos en esta tarea permite detectar algunos otros rasgos que resaltan la diferencia entre lo que serían los resúmenes elaborados para fines personales de aprendizaje o retención, así como de los que son parte de escritos o informes académicos. No obstante esto, los textos del corpus presentan algunos de los rasgos compositivos más típicos del resumen entendido como género 'escolar', por cuanto suponen la orientación reformulativa con tendencia a la reproducción del TF. Desde el punto de vista que seguimos, los rasgos típicos del resumen pueden esquematizarse en una tendencia a la reformulación parafrástica reductiva de un TF, junto con la adopción de rasgos equivalentes a los de dicho texto. Esta caracterización nos facilita un modelo de descripción de las correspondencias entre el TF y el texto producido, lo que nos parece de gran utilidad para el estudio selectivo del PDN, sin eliminar la concepción interactiva del trabajo con el texto propio y con el texto de otro, como un supuesto del proceso de composición. La reformulación posibilitaría el establecimiento de equivalencias con mayor o menor grado de continuidad con el TF. 
De las distintas posiciones acerca de la reformulación, nos valemos, en este caso, principalmente, de las que conciben una equivalencia del texto propio con otro (Fuchs, 1994) antes que de las que la conciben como actividad de reescritura. En esta dirección, Fuchs (1994) permitiría contemplar las especificidades de la reformulación como un determinante fuerte de los principios que rigen la selección y el uso de la unidad en el contexto que nos ocupa. La autora se refiere a dos grandes tipos de reformulación que son la reformulación explicativa y reformulación imitativa. La primera busca reconstruir y explicitar el sentido del TF, mientas que la segunda se centra en la reproducción del TF. Los resúmenes en el contexto educativo tienden a reflejar más el segundo tipo de reformulación. Gülich y Kotschi (1983, 1995), por su parte, colaboran en la clasificación de la reformulación parafrástica que resultaría el tipo de estrategia equivalente a los mecanismos de reformulación distinguidos por Fuchs (1994). Las estrategias que guían la reformulación parafrástica en la composición del resumen serían compatibles con las caracterizadas por otros autores en modelos más dependientes del perfil de procesamiento de cognitivo de la estructura del texto (van Dijk, 1980, 1983).

\section{Marco metodológico}

\subsection{Hipótesis y supuestos específicos del estudio}

Los supuestos guías de esta investigación se apoyan en el marco de referencia que liga las condiciones de producción textual con determinantes genéricos y con perfiles funcionales dependientes de la tarea y las prácticas, en este caso curriculares, dentro de una comunidad profesional y académica. Por tratarse de una síntesis-resumen, interpretamos las determinantes genéricas y textuales que derivan de la propensión reformuladora reproductiva del TF. La orientación compositiva del texto del corpus haría posible un seguimiento más preciso de las operaciones compositivas de cada escritor, así como de la selección de recursos y mecanismos reformuladores que son incorporados al texto final, a la vez que permite suponer operaciones y principios puestos en juego en la composición del texto que pueden favorecer el uso del PDN, independientemente de otras condicionantes.

Como parte de nuestro marco interpretativo, partimos de suponer que en textos reformulativos de síntesis o resumen escolar, las operaciones compositivas del escritor que comprenden el uso del demostrativo admitirían caracterizarse por:

a. el grado de autonomía relativa con que el escritor hace uso de la unidad en relación con el TF;

b. el grado de preferencia por la selección de este recurso para favorecer la continuidad temática; 
c. un mayor o menor compromiso en el uso del demostrativo con el contenido semántico conceptual y/o con relaciones argumentativas, en oposición a funciones más híbridas, inespecíficas o demarcativas;

d. el tipo de compromiso interactivo que el escritor establece con la elaboración del texto producido, en donde la comprensión de la fuente puede considerarse condicionante de la producción del texto finalmente producido. Este ítem se puede vincular al grado de esfuerzo, operacionalizable por el tipo de estrategias recurrentes para la composición de la síntesis-resumen.

En función de lo anterior, sería posible identificar aquí problemas adicionales en el seguimiento de la referencia anafórica, aspecto que tratamos con mayor detalle en otro trabajo. Cabe agregar, por último, que no es posible dejar de lado las tensiones entre principios funcionales que pueden ser más dependientes del género y que se manifiestan a través de la selección de recursos compositivos. En el caso del resumen, la tendencia a la abreviación y a la conservación del contenido son principios altamente relevantes y en pugna. El PDN puede acomodarse para servir fácilmente a ambos principios, aunque esto solo puede inferirse de las categorías de uso, ya que, de por sí, la función anafórica sustitutiva se aviene a la economía de recursos.

\subsection{Participantes}

Se recogieron producciones escritas de 65 estudiantes universitarios de Psicología de la Facultad de la Universidad de la República, Uruguay. Los estudiantes de la muestra contaban con una media de edad de 25 años. Se distribuyeron por sexo en 11 varones y 54 mujeres.

\subsection{Diseño de la tarea}

Se recogieron en total 65 síntesis escritas realizadas domiciliariamente. El marco en el que se solicitó la tarea para la obtención del corpus fue el de un curso del tercer año de la Licenciatura. La consigna de la tarea consistió en el pedido de que elaboraran la síntesis en base a un texto que se les entregaba de no más de 5 páginas, para la cual no se daban indicaciones sobre cómo proceder para su realización, ni se planteaban limitaciones al uso de fuentes y otros recursos. La consigna fue informada oralmente y se entregó por escrito. En ningún caso se dieron criterios de elaboración de la síntesis. Se informó que la tarea sería evaluada cualitativamente, sin calificación. Se seleccionaron los textos de estudiantes que habían trabajado con uno de los textos que se habían distribuido en toda la generación de cursantes.

\subsection{Descripción del corpus y materiales}

Se empleó como TF un fragmento correspondiente a los primeros apartados de un capítulo de J. Bruner de la obra 'Actos de significado'. El material seleccionado como 
estímulo para la elaboración de la síntesis estuvo compuesto de 3.350 palabras. El texto asignado es de carácter básicamente expositivo.

\subsubsection{Procedimientos}

a. Como primer paso se segmentó el texto de la fuente en párrafos o unidades menores relativas, según la extensión del párrafo.

b. En el corpus estudiantil se consignó la frecuencia de ocurrencia de los demostrativos próximos a la unidad seleccionada, determinantes ('este', 'esta', 'estos', 'estas') y pronominales ('est/e/a/os-as', 'esto’). Además se relevó para fines complementarios el caso de los demostrativos neutros 'ese/a/o' y 'aquello/o', y sus correspondientes plurales.

c. Se seleccionaron las ocurrencias del pronombre neutro 'esto' y se estudió el contexto sintáctico y semántico, así como las funciones que desempeñaba la unidad en la secuencia.

d. Cada párrafo en el que aparecía el pronombre demostrativo se comparó con el TF a través de tres grandes series de categorías.

i. Las que indicaban la distancia reproductiva de la fuente.

ii. Las que clasificaban el tipo de contexto de ocurrencia del pronombre y la operación de reformulación en relación al TF.

iii. Las que indicaban el rol funcional.

Estas series se aplicaron a todas las ocurrencias presentes en el corpus.

Para i. se identificaron las ocurrencias del 'esto', a los efectos de diferenciar las ocurrencias debidas a la reproducción por copia del texto original en los textos estudiantiles.

e. Por último, se buscó identificar las relaciones semánticas y pragmáticas de los segmentos vinculados con la ocurrencia del 'esto', sin descuidar la relación con el TF, a los efectos de obtener un perfil funcional del PDN en el texto reformulado. A partir de esto, proponemos clasificación de los tipos de usos.

\subsection{Categorías de análisis}

\subsubsection{Análisis de los contextos gramaticales del PDN}

Se analizaron los contextos sintácticos y semánticos, discriminando antecedentes y segmentos pospuestos. En aquellos casos en que no se trataba de una reproducción estricta de la fuente ${ }^{1}$, se realizó un análisis sintáctico del contexto de ocurrencia del demostrativo neutro 'esto' en relación al TF y al texto producto de la reformulación. 
Para ello se tomó como norma la exclusión de los segmentos basados en la copia de los segmentos antecedentes y de la ocurrencia de la propia unidad. Se incluyeron para el análisis, sin embargo, aquellos casos en que solo se copiaba el demostrativo y el segmento pospuesto y no el antecedente. Para la clasificación básica del rol gramatical se tomaron en cuenta la posición sujeto, frase preposicional y objeto directo.

\subsubsection{Distancia reproductiva del TF}

Se clasificaron las ocurrencias del 'esto' en usos más independientes y distanciados de la fuente y usos manifiestamente dependientes de dicho texto. Para establecer mejor estas relaciones, se diferenciaron los contextos del 'esto' que tendían a la repetición o reproducción literal del TF respecto de aquellos que mostraban una tendencia reformuladora parafrástica o una versión más distanciada de la guía compositiva de la fuente. Cada una de estas categorías se aplicó al contexto antecedente y pospuesto del 'esto', así como a su propia ocurrencia. Se distinguió un tipo de reproducción 1, que equivaldría a la reproducción estricta (Fuchs, 1994), caracterizada por ser una versión literal del TF, sin marcación de citación, y una reproducción 2, que solo se diferenciaba del segmento de la fuente por cambios mínimos que conservaban la estructura gramatical. Para todos los demás casos, se determinó la categoría de reformulación parafrástica.

\subsubsection{Categorías del contexto de ocurrencia del 'esto' y de reformulación del TF}

Ya que partimos del supuesto que la base de la composición del texto estudiantil es la reformulación del TF, proponemos una clasificación de las ocurrencias que toman en cuenta esta relación. Para su clasificación se atendió a las operaciones de reformulación que contenían el demostrativo en su relación con el TF en el que se basaban los escritos estudiantiles. Las siguientes categorías de análisis se confeccionaron para estudiar estos distintos usos.

\subsubsection{Reproducción de la ocurrencia que aparece en el original}

Esta categoría se consignó cuando había repetición o copia de la unidad en un contexto semejante al del TF.

\subsubsection{Agregado a la paráfrasis del TF}

Se consignó cuando el contexto que contiene el 'esto' se identificaba como equivalente sintáctica y semánticamente de segmentos del TF, sin que el TF contuviera la unidad.

\subsubsection{Sustitución}

Cuando se sustituye un sintagma del TF por un segmento que incluye el 'esto'. Este caso se identifica cuando hay una relación de correspondencia entre el TF y el 
texto producido por el estudiante que incluye el pronombre demostrativo. Esto se da cuando una FN o FV del TF corresponde a un segmento del texto producido que incluye el 'esto' y que, por lo tanto, el pronombre aparece sustituyendo la frase del TF, sin necesaria reconstrucción del texto producido. Por ejemplo, "Esta famosa historia se puede dividir en dos partes" $\rightarrow$ "Esto se puede dividir en dos partes."

\subsubsection{Independencia compositiva del TF}

Se determinó en los casos en que no era posible establecer equivalencias parafrásticas entre el TF y el texto de la síntesis. Esta falta de equivalencia sería relativa a la ocurrencia del neutro, así como al antecedente y al segmento pospuesto que lo contiene.

\subsection{Rolfuncional del PDN}

El análisis del rol funcional se compone de la propuesta de categorías para su análisis. Las categorías se proponen como resultado del conjunto del análisis y pretenden contemplar aquellas funciones conectadas a la hetero-reformulación, así como las que son más características del PDN en sus distintos contextos gramaticales.

\section{Resultados}

\subsection{Distribución de los demostrativos, determinantes y pronominales}

El conjunto de los textos que integran el corpus presenta una media de 546 palabras. El número de palabras presentó un rango máximo de 1.765 palabras y un mínimo de 129. Los textos extensos tanto como los de menor número de palabras mostraron ocurrencias del pronombre neutro. Se puede presumir que, para este estudio, la diferencia en extensión no es significativa ${ }^{2}$. De los 65 textos que integraron el corpus inicial, el 77\% presentó alguna ocurrencia del demostrativo neutro 'esto', por lo que la muestra quedó compuesta por 50 textos. Ubicamos en las Tablas 1 y 2 la distribución de frecuencias de los demostrativos determinantes y los pronominales que son morfológicamente semejantes en el total de la muestra $(n=50)$.

Tabla 1. Frecuencias y porcentaje de determinantes.

\begin{tabular}{|l|c|c|c|c|c|}
\hline Determinante & 'este' & 'esta' & 'estos' & 'estas' & total \\
\hline Suma & 74 & 92 & 42 & 28 & 236 \\
\hline$\%$ & 31 & 39 & 18 & 12 & 100 \\
\hline
\end{tabular}


Tabla 2. Frecuencias y porcentaje de pronombres demostrativos.

\begin{tabular}{|l|c|c|c|c|c|c|}
\hline Pronombre & 'esto' & 'este' & 'esta' & 'estos' & 'estas' & total \\
\hline Suma & 87 & 18 & 45 & 42 & 14 & 206 \\
\hline$\%$ & 42 & 9 & 22 & 20 & 7 & 100 \\
\hline
\end{tabular}

Los demostrativos pronominales no neutros siguieron la norma predominante de referirse a unos pocos sustantivos que coincidían con núcleos temáticos del TF. Como se puede apreciar, el pronombre 'esto' supera ampliamente la ocurrencia de los demostrativos considerados individualmente. En la Tabla 3 presentamos la comparación entre los determinantes, los pronominales no neutros y el neutro.

Tabla 3. Comparación de demostrativos determinantes y pronominales.

\begin{tabular}{|c|c|c|c|c|}
\hline Demostrativo & Determinantes & PDem No-N & PDem Neutro & Total \\
\hline Suma & 236 & 119 & 87 & 442 \\
\hline$\%$ & 53 & 27 & 20 & 100 \\
\hline
\end{tabular}

En el total, los determinantes y la suma de los pronombres demostrativos similares morfológicamente, neutros y con variación de número y género, tienen una frecuencia relativamente equivalente. Aislando la relación entre pronombres demostrativos no neutros y neutros se obtiene que, los primeros, aparecen un 15\% más que los neutros, diferencia que no resulta significativa. No incluimos en la tabla los otros pronombres neutros demostrativos, pero su ocurrencia fue insignificante en relación a los de esta serie.

\subsection{El pronombre demostrativo neutro 'esto' en el corpus}

La media de ocurrencias del demostrativo 'esto' por texto fue de 2 en el total de la muestra; el máximo de ocurrencias en el texto de la síntesis no superó los 4. La proporción de textos que presentaron más de una ocurrencia fue del $42 \%$. El total de ocurrencias detectadas en el conjunto del corpus $(n=50)$ fue de 87 . De los 18 párrafos que contiene el texto de la fuente, los párrafos de las síntesis estudiantiles que contienen la unidad estudiada fueron 15 del total.

Se consignó una marcada preferencia por el empleo del demostrativo neutro en correspondencia con algunos segmentos del texto de referencia. El uso predilecto del demostrativo se dio en relación con 5 del total de párrafos en los que se segmentó el TF. Este uso preferencial se verificó en el 78\% del total de las ocurrencias. Los segmentos que contaban con mayor número de ocurrencias del pronombre fueron los ubicados al comienzo del TF; solo en un caso el segmento de referencia se ubicaba en la mitad de texto. 


\subsection{Funciones gramaticales y clasificación funcional}

El 100\% de los demostrativos contaron con valor anafórico entendido en sentido amplio y no solo como correferencia puntual de un SN. En cuanto a las funciones gramaticales que cumplieron en el texto, encontramos: un 43\% correspondiente a la función anafórica como sujeto, $33 \%$ a objeto directo y $23 \%$ a frase preposicional. Destacamos el uso del 'esto' en posición sujeto u objeto directo. Este fue el caso de más el $76 \%$ de las ocurrencias. Con respecto a otras construcciones consignadas, se detectó un $8 \%$ de formulaciones pronominales del demostrativo precedidas por el cuantificador universal 'todo': 'todo esto', con carácter demarcador en un 8\%, y con valor enfático en 13\% (por ejemplo: “tenemos la creencia de que esto que creemos”). En los casos del valor demarcativo, sobre todo expresado a través del cuantificador 'todo', la referencia se propone en un bloque indeterminado, evitando el compromiso con el establecimiento de una especificación que no parece pretenderse.

\subsection{Distancia reformulativa del TF y operaciones de reformulación}

En el Gráfico 1 se aprecia el porcentaje de distribución de las categorías definidas en 2.5.3 en el total de ocurrencias ( $n=87$ ). Como se aprecia en el Gráfico 1 , los usos mayoritarios correspondieron a las formulaciones que se categorizaron en 2.5.3.2 como 'agregado' y, por lo tanto, asociadas parafrásticamente a la reformulación de algún segmento textual de la fuente. El segundo uso mayoritario correspondió a la reformulación sustitutiva del TF por parte del estudiante. Encontramos que un 17\% del total de las ocurrencias correspondieron a la reproducción estricta del TF, es decir, la reformulación de tipo 1 , y un $66 \%$ correspondió a una reformulación parafrástica más o menos amplia, reformulación de tipo 2 ('sustitución' + 'agregado'). La categoría de mayor distanciamiento del TF (independencia del TF) se dio solo en un 17\% del total.

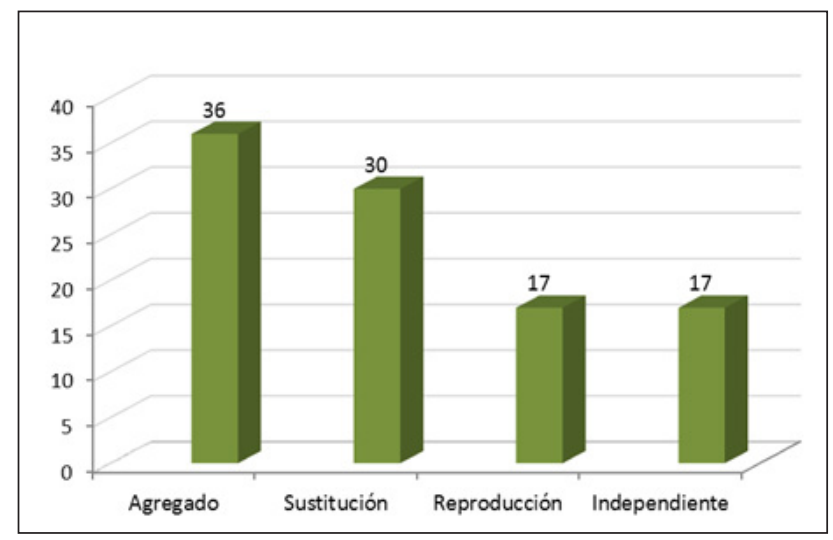

Gráfico 1. Porcentaje de ocurrencias del PDN relativas al TF $(\mathrm{n}=87)$. 
Siguiendo a Fuchs (1994), consideramos que el tipo de reformulación predominante en nuestro corpus sería el de la paráfrasis imitativa que se incluiría dentro de la reformulación parafrástica reductiva de Gülich y Kotschi (1983).

\subsection{Uso y funciones del PDN}

\subsubsection{Categorías de uso definidas}

A continuación proponemos una serie de categorías en las que clasificamos las distintas ocurrencias del pronombre neutro 'esto', atendiendo a las relaciones semántico-pragmáticas en las que se insertaba y la relación con el TF. La confección de las categorías toma en cuenta el tipo de texto y las operaciones de reformulación compositiva de otro texto, específicamente, al catalogar el uso de las ocurrencias del PDN. Concebimos las siguientes categorías que abarcan los contextos de ocurrencia del 'esto': copia, enlace, reinterpretación del discurso del autor del TF, resuntivos, reflejos, organizadores y argumentativos o inferenciales.

Consignamos que algunas categorías no son excluyentes por lo que una ocurrencia podría ser caracterizada en más de un caso. Recurrimos a la ejemplificación en las categorías que no son inmediatamente interpretables, con los textos estudiantiles, y cuando así lo requiere la categoría, colocamos el TF que sirvió de base a la formulación.

a. Copia o reproducción. Reproduce segmento del texto fuente que incluye el 'esto's.

b. Enlace. En esta categoría incluimos los usos que calificamos de mero 'enlace' entre partes del texto, de modo tal que se convierte en una fórmula neutralizada que simplemente conecta segmentos en el discurso que están sugeridos por la línea argumental del TF. En algunos casos el 'esto' separa segmentos que forman parte de una única secuencia en el TF. Por ejemplo en (1):

(1) "Hay una división entre un mundo de experiencia y un mundo, que es autónomo respecto a la experiencia. Esto crea tres dominios, que cada uno requiere una forma distinta de interpretación".

El texto subrayado resulta una reproducción del TF; el 'esto' simplemente sirve a la continuidad del texto sin compromiso significativo entre las partes más allá de la que está reproducida.

c. Reinterpretación del discurso del autor del TF. Siguiendo la línea argumental del TF, el autor del texto reformulado expande, explicita o reinterpreta el sentido de la expresión autorial metadiscursivamente. El ejemplo (2) podría caracterizar este uso.

(2) "Los seres humanos hacen cosas basándose en sus creencias y deseos. Esto para Bruner moviliza toda la vida". 
d. Resuntivo. Hace referencia inespecífica a argumentos referidos antes, operando como una forma de 'encapsulamiento' del antecedente. Generalmente, estos suelen ir acompañados del cuantificador 'todo' que refuerza el sentido amplio y resuntivo de la anáfora de una amplia referencia. Esto se ve en el ejemplo (3).

(3) "Como parte final el autor habla del YO de psicología popular y la diferencia que se da en diferentes cultura, en una donde no a sido alfabetizado y la otra sí (...) Expresa esto por las críticas que se realiza a la psicología popular...”.

e. Reflejos. Este caso supone que el movimiento del autor del TF queda 'reflejado' en el texto reformulado, a través de sustituciones o equivalencias que mantienen el giro argumentativo y expresivo del autor, mediante una reformulación parafrástica reductiva. Seleccionamos los ejemplos (4), (5) y (6).

\begin{tabular}{|l|l|}
\hline \multirow{2}{*}{ Texto fuente } & $\begin{array}{l}\text { "...el concepto fundamental de la psicología humana es el de signifi- } \\
\text { cado y los procesos y transacciones que se dan en la construcción de los } \\
\text { significados. Esta convicción se basa en dos argumentos relacionados } \\
\text { entre sí...." }\end{array}$ \\
\hline \multirow{3}{*}{ Texto estudiantil } & (4) "Esto lo argumenta diciendo que..." \\
\cline { 2 - 2 } & (5) "Esto, a su vez, se basa en dos argumentos relacionados entre sí ..." \\
\hline & (6) "Para esto se basa particularmente en dos nociones..." \\
\hline
\end{tabular}

En el primer segmento, la mayoría de los usos del pronombre demostrativo corresponden a la sustitución parafrástica del TF, en donde se incorpora el 'esto' y se reformula parafrásticamente el segmento postpuesto que corresponde al de la fuente. La sustitución operada por el texto meta cambia la FN del TF, por ejemplo: "esta convicción se basa" del TF, se cambia por una expresión que incorpora el demostrativo neutro, ejemplos (4), (5) y (6). En estos casos la reformulación reproductiva tiende a su grado máximo de equivalencia por omisión de una $\mathrm{FN}$ e intercambio de demostrativos, esto es, que la operación va del demostrativo definido en el SN del TF hasta su sustitución por la fórmula pronominal.

Los siguientes casos (7), (8), (9) son también claros ejemplos de esta categoría.

\begin{tabular}{|l|l|}
\hline \multirow{2}{*}{ Texto fuente } & $\begin{array}{l}\text { "Esto es el esqueleto desnudo de los argumentos a favor de lo que he } \\
\text { denominado 'psicología cultural', ..." }\end{array}$ \\
\hline \multirow{3}{*}{ Texto estudiantil } & (7) “A esto le llamó "psicología cultural”..." \\
\cline { 2 - 2 } & $\begin{array}{l}\text { (8) “Todo esto le permite la creación de lo que denomina "psicología } \\
\text { cultural"..." }\end{array}$ \\
\cline { 2 - 2 } & (9) "Esto es lo que denomina "psicología cultural"..." \\
\hline
\end{tabular}


Aquí se da la reformulación del $\mathrm{SN}$ del TF con repetición del verbo de la fuente o su cambio por otro con un valor semántico aproximado. En algunos casos, se observa que la variación equivalente supone una pérdida del nivel de especificación que se da en el TF, presumiblemente, como forma de adaptación a la formulación más propia del estudiante. Este es el caso de la sustitución de la frase "dos argumentos" del TF, por "dos nociones".

En los distintos ejemplos es bien claro el establecimiento de la equivalencia semántica entre el 'esto' del nuevo texto y el $\mathrm{SN}$ de la fuente. El paralelismo resulta respaldado por la copia del verbo del original o el cambio por una selección léxica aproximada. En estos ejemplos, la operación de reformulación que incluye el pronombre demostrativo, supone una mera operación de abreviación de un valor referencial ya dado por el texto de la fuente, por lo que el uso de la unidad podría catalogarse como de 'bajo riesgo'. En ninguno de estos ejemplos el estudiante se juega a proponer variaciones significativas del contenido semántico, lo que sugiere un escaso nivel de reformulación interpretativa que trascienda la equivalencia superficial entre el texto de la fuente y el texto producido o meta. Así, se asegura una correspondencia entre el segmento antecedente y el pospuesto que contiene la emergencia de posibles dificultades en relación con usos más libres.

f. Organizadores. Los que se orientan a separar segmentos en función del orden del texto y de la selección del autor TF. Generalmente, se acompañan de marcadores de ordenación 'antes de esto', 'esto último', 'a partir de esto'. La función demarcativa del PDN, referida por algunos autores, puede verse en relación con este uso.

g. Argumentativos o inferenciales. En este contexto el 'esto' sirve al vínculo entre segmentos a través de una operación inferencial, generalmente conclusiva o causativa que ubica el antecedente como un término para expresar un argumento razonado. Uno de los ejemplos encontrados en el corpus es el siguiente:

(10) "El texto trata el tema de la Psicología popular, considerándola a la misma como un instrumento de la cultura, con el cual ésta moldeará la vida y la mente humanas. Es por esto que la Psicología humana debe abordar la cultura y el significado".

\subsubsection{Síntesis de resultados}

La Tabla 4 sintetiza el comportamiento general del 'esto' en la relación a las categorías anteriormente expuestas en el total de las ocurrencias del PDN ( $\mathrm{n}=87$ ). Los usos más frecuentes fueron los que se ubican en la categoría 'reinterpretación de la palabra del autor', 'resuntivo', 'enlace', 'reflejo' y 'copia'. 
Tabla 4. Frecuencias y porcentajes de categorías del 'esto' en el corpus ( $n=87)$.

\begin{tabular}{|l|c|c|c|c|c|c|c|c|}
\hline Enlace & Resuntivo & Argument. & Reflejo & $\begin{array}{l}\text { Reinterpre- } \\
\text { tación de la } \\
\text { palabra del } \\
\text { autor }\end{array}$ & Copia & Organiz. & Total \\
\hline Suma & 13 & 10 & 6 & 15 & 23 & 15 & 5 & 87 \\
\hline$\%$ & 15 & 12 & 7 & 19 & 24 & 17 & 6 & 100 \\
\hline
\end{tabular}

Encontramos un escaso porcentaje del 'esto' con valor conectivo de carácter argumentativo. En estos usos se introduce una pretensión de inferencia o de razonamiento. Se identificaron dentro de esta categoría valores de finalidad o causalidad: 'para esto' y 'por esto', con valor consecutivo. Los usos más intelectualizados dentro del texto estudiantil correspondieron a esta categoría. El porcentaje de ellos es muy bajo, además, 4 de ellos están sugeridos por el TF. Es interesante hacer notar que la categoría de reinterpretación del movimiento del autor del TF a la que sirve la anáfora con 'esto', es una categoría diferente de la que se observa en los resúmenes de uso personal, lo que contribuye a diferenciar estos textos por la intencionalidad comunicativa que ponen de manifiesto y que revela un contexto de tarea y finalidad diferenciado.

\section{Discusión de los resultados}

Como podíamos esperar, en el corpus, el grupo de los pronombres demostrativos de base 'est-' (neutros y no neutros) predominaron sobre la ocurrencia individual de los otros pronombres demostrativos neutros referidos por la RAE (2010). Se destaca que el PDN alcanzara una proporción tan alta en comparación con las formas pronominales no neutras, lo que refuerza el supuesto de que esta forma pronominal puede ser una fórmula preferida, al menos en textos semejantes producidos por escritores nóveles. Atribuimos esta preferencia, precisamente, a la maleabilidad que ofrece en su función anafórica por la amplitud y posible vaguedad de su conexión, destacada por varios autores. Resultó interesante consignar su comportamiento en relación a textos reformulativos como la síntesis-resumen, ya que por la estrategia de composición que se sigue para su elaboración, todo el texto estudiantil puede considerarse que está en 'función de referencia' con el TF.

Como se ha planteado antes, el modelo guía que oficia el TF parece facilitar un seguimiento más preciso de las operaciones compositivas del escritor y de la selección de las unidades o recursos que son incorporadas al texto final. Estas operaciones estarían vinculadas con la comprensión del TF y con la ‘conversión’ que debe efectuar el escritor para la composición de su texto. Por tratarse de un texto básicamente de carácter hetero-reformulativo, se hizo posible inferir más específicamente ciertas operaciones vinculadas a la interacción entre la lectura del TF y la composición del propio, en donde se incorporaba el demostrativo neutro 'esto'. 
La puesta en comparación del texto producido con el TF a los efectos de estudiar el PDN permitió observar un tipo de usos sustitutivos del pronombre, que se podría recomponer fácilmente a partir del enlace con el texto de la fuente, oficiando un señalamiento a los contenidos y a la estructura sintáctico-gramatical de los segmentos de dicho texto. Siguiendo a algunos autores (Lyons, 1977; Diessel, 1999; RAE, 2010), este uso sugiere una función que podría calificarse de híbrida o más próxima al carácter deíctico de la expresión, puesto que parece apuntar al TF.

\subsection{Estrategias y principios predominantes en la composición}

En relación al corpus, ordenamos los siguientes aspectos que permiten relacionar el uso del PDN con el TF y su contexto genérico y situacional.

a. la estrategia compositiva global del texto en relación al TF;

b. las estrategias y operaciones predominantes en la producción de la ocurrencia;

c. los principios orientadores de la composición y de las operaciones de reformulación del TF;

d. implicancias del relacionamiento de los estudiantes con los textos.

Seleccionamos, a continuación, algunos aspectos destacados que permiten detenernos en cada uno de estos puntos a partir de los resultados obtenidos.

\subsubsection{Estrategia compositiva global del texto}

Como ya había sido comprobado en otros trabajos relativos a un corpus semejante, en el actual corpus, consignamos como estrategia predominante de producción la reformulación reproductiva de otro texto, que es una estrategia típica de la elaboración de resúmenes o síntesis en contextos educativos. Los estudiantes seleccionaron preferencialmente algunos de los segmentos del TF como motivo de reformulación, lo que facilitó la comparación entre la producción más libremente orientada y la más regida por la fuente, al minimizar la dispersión de la base sintáctica y semántica del texto producido. Los estudiantes tendieron a modificar el texto de la fuente por paráfrasis y sustitución de segmentos relativos a la fuente. La estrategia compositiva general expresó una tendencia mimética, por la cual el texto producido se diferenciaba muy poco de la fuente. Esta tendencia se hizo manifiesta en la reproducción estricta del TF o en su reformulación parafrástica a lo largo de cada síntesis.

\subsubsection{Estrategias y operaciones predominantes en el uso del 'esto'}

Se destacó la tendencia al uso de este pronombre demostrativo en contextos reformulativos parafrásticos, antes que en contextos de elaboración más independientes de la fuente o de su literalidad. El porcentaje mayoritario de ocurrencias correspondió al método de reformulación, en donde el estudiante se distancia mínimamente del texto guía. En el 17\% del total de ocurrencias, esta estrategia general llegó a incluir 
la unidad pronominal del TF considerada, así como a sus contextos antecedente o pospuesto. No obstante, en el mayor porcentaje de ocurrencias, el 'esto' se incorporó como recurso cohesivo diferenciándose de la reproducción directa del TF. Los bajos porcentajes de las funciones argumentativas o metadiscursivas, que serían las más afines a un contexto distanciado de la función modélica del TF, concurren en la misma dirección.

Las operaciones predominantes de incorporación del demostrativo 'esto' fueron la réplica o sustitución de frases del TF por dicha unidad, o bien su agregado en contextos controlados de reformulación en donde adquiría el valor de nexo inespecífico en cuanto a la delimitación o especificación del contenido al que hacía referencia como anáfora. Encontramos usos anafóricos con una referencia poco especificable en relación con el contexto semántico y conceptual antecedente del texto estudiantil. Como hemos mencionado, la bibliografía sobre el tema (Moskovit, 1983; Geisler et al., 1985; Finn, 1995) señala que ciertos usos del pronombre favorecerían la producción de efectos en el lector asociados con la imprecisión y vaguedad de la referencia. En nuestro caso, confirmamos que la continuidad anafórica ofrecida por este pronombre demostrativo puede calificarse de laxa y al mismo tiempo débil.

Las operaciones psicolingüísticas inferibles sugieren que los usos predominantes detectados del demostrativo comprometieron poco el proceso analítico de trabajo con el TF, dimensión que estaría involucrada en cualquier abordaje profundo de la comprensión y producción del texto. Los usos más frecuentes demostraron un rebajamiento del esfuerzo puesto en la selección y en la accesibilidad del segmento con información semántica que operaba como antecedente.

\subsubsection{Dependencia genérica y tensiones entre principios de producción}

Partimos de suponer que el contexto genérico que caracterizaba al corpus favorecía ciertas operaciones cognitivas y textuales por parte del escritor que permiten estudiar más a fondo peculiaridades del género textual, tanto como del involucramiento del escritor en su relación con el texto leído y con el texto producido. La dependencia genérica se confirmó ampliamente en los textos del corpus que pudieron ser categorizados como síntesis-resumen. Los textos presentaron las características típicas del resumen según distintos autores, pero admitieron también la presencia expresa de marcadores metadiscursivos y reformuladores de la palabra del autor del TF, que no son típicos del resumen para uso personal. Esta diferencia con los resúmenes típicos realizados para fines de estudio o aprendizaje hace más interesante la identificación y el seguimiento de la preferencia compositiva relativa a la unidad pronominal.

A esto puede agregarse la idea de que el TF planteó determinadas restricciones a las operaciones de selección del escritor a la vez que facilitó la redacción del propio texto. En el tipo de texto producido la ocurrencia del 'esto' tuvo menos oportunidades de manifestarse liberada de los principios de producción que son relativos al tipo 
genérico que favorecen la tendencia reproductiva. Paradójicamente, sin embargo, por ser este el soporte oficiado por el TF, pudo verse incrementada la suspensión de la responsabilidad sobre el proceso de composición que selecciona los segmentos y los interpreta. De este modo, la guía del TF puede promover una tendencia a relajar el compromiso con las relaciones semánticas expuestas. El contexto de actividad que acompañó su realización podría incidir para agudizar más esta propensión.

Puede suponerse que la introducción compositiva exitosa del pronombre demostrativo 'esto' en el texto estudiantil debería cumplirse atendiendo a una doble orientación, por un lado, la tendencia a la 'abreviación' del TF y, por otro, la de conservación de las relevancias semántico-conceptuales y argumentativas del TF. De estas orientaciones resultan dos de los principios inherentes a la elaboración del resumen-síntesis, como tipo de género determinante de la producción que deben ser puestos en correspondencia con las condiciones prácticas para cobrar sentido. De acuerdo con nuestros resultados, los textos estudiantiles mostraron la tendencia clara a la 'abreviación', mientras que la conservación de relevancias semántico-conceptuales en la reelaboración escrita tendió a cierta degradación de las relaciones. La economía de recursos empleados en la producción textual y la tendencia a la abreviación del TF parecieron ser principios rectores del conjunto de las estrategias de producción de la gran mayoría de los textos que integraron el corpus. Una comprobación indirecta de esto la podemos inferir de la preferencia de valerse de los primeros párrafos del TF para producir el propio, con escasa integración del resto del material, además de las categorías prevalentes en el análisis del uso del PDN en los textos.

Por último, cabe señalar aspectos del contexto de realización del texto. Parece previsible que el hecho de que los escritos del corpus respondieran a un requisito de evaluación curricular, facilitara un control más definido y abarcativo de la producción escrita que la de los contextos funcionales más asociados al uso personal (Torres, 2004, 2009a, 2009b). Las condiciones curriculares de producción del texto estudiantil tenderían a plantear una pretensión retórica y comunicativa más orientada por la claridad y la objetividad del texto producido. Por su parte, el TF que seleccionamos es catalogable dentro de los géneros discursivos académicos de carácter expositivo y argumentativo, que presentan la particularidad de contener varias secuencias reformulativas expansivas y repetitivas de los argumentos centrales, lo que le otorgaría un carácter más ‘didáctico’ y facilitador para el lector. Es posible señalar, sin embargo, que la síntesis elaborada fue de carácter domiciliario y no tenía calificación cuantitativa, lo que puede haber favorecido que la elaboración de las mismas haya contado con una incidencia mayor de factores distractivos, así como con un rebajamiento del nivel de esfuerzo debido a la presumible falta de sanción calificatoria de su producción. Las características que asumieron las producciones se pueden asociar, en este sentido, con una actitud menos comprometida con la elaboración autónoma del texto que se traduciría en una preocupación menor por efectuar procesos de revisión y/o relectura reformulativa del texto. Como sabemos, estas estrategias constituyen recursos básicos 
para mejorar la calidad de la escritura y de la comprensión (Bereiter \& Scardamalia, 1987; Flower, Stein, Ackerman, Kantz, McCormick \& Peck, 1990; Higgins, Flower \& Petraglia, 1992).

Como resultado del análisis se plantea por esto la interrogante de cómo la tarea y la orientación genérica del texto producido por los estudiantes pueden haber contribuido al uso de este demostrativo pronominal proporcionando antecedentes y segmentos pospuestos reformulables imitativa o reductivamente a partir del TF.

\subsection{Implicancias de la relación de los estudiantes con los textos}

A los efectos de aportar a la significación global del estudio, resulta interesante apreciar posibles orígenes y consecuencias que relacionen los resultados de los textos leídos y producidos por los estudiantes. El corpus analizado se conecta con prácticas y con un tipo de tarea y consigna típica de entornos educativos, ya que la producción se realizó en un marco institucional y en el contexto de una actividad curricular, relativamente regular, aunque no fuera instruida. El texto del corpus seleccionado fue el de una síntesis muy próxima a la realización prototípica de un resumen. El resumen es guiado en su realización por un TF con respecto al cual las operaciones de reformulación parecen someter el conjunto de los recursos compositivos que se ponen en juego en la producción. El tipo de texto permite hacer alusión a tres aspectos en relación con nuestro estudio y el contexto situacional que abarca la tarea: a) la competencia compositiva y gramatical; b) la especificidad genérica; y c) la dimensión de la tarea.

a. El hecho de tratarse de un texto producido en base a la reformulación de otro podría considerarse un facilitador de una producción regulada por el modelo de texto, sin embargo vimos que esto no está exento de problemas, ya que los estudiantes tendieron a componer su texto mediante un tipo de paráfrasis muy restrictiva. Teniendo en cuenta el texto producido, no es posible dejar de considerar que en la prosa estudiantil, el uso del PDN puede alimentarse de debilidades vinculadas al manejo de la escritura y de los procesos de composición, como aquellas que derivan del nivel gramatical y del proceso de textualización en general. La producción de efectos tales como la imprecisión y la vaguedad podrían verse como consecuencia de esta situación, independientemente del uso del demostrativo. La comprensión del TF sobre la que se basa, el proceso de composición y la competencia en el género discursivo y en la escritura formal académica en general deben hacerse notar como variables. El uso del pronombre neutro se vincula al tratamiento de relaciones intratextuales más o menos específicas a los tipos de textos estudiados por lo que, notoriamente, dependerá su apropiada inclusión compositiva del relacionamiento del estudiante con la escritura y los textos expositivos y argumentativos en general.

b. La frecuencia de producción de resúmenes para fines de aprendizaje, retención y/o comprensión de contenidos a través de otros textos, sugiere el interés que 
puede tener estudiar las operaciones y estrategias compositivas de los estudiantes en casos similares, ya que la lectura y la comprensión activa o residual se ponen en juego a través de la reformulación de otro texto. Hemos hecho notar, sin embargo, que si bien el corpus responde a rasgos prototípicos del resumen, no se confunde con las características que adopta el resumen para los fines personales de aprendizaje. Esto se explica por el propio origen funcional y el destino comunicativo del texto obtenido en función de la tarea asignada, aspectos que promoverían una producción mayormente controlada, aunque luego esto esté sujeto a otras condicionalidades. De cualquier manera, en uno u otro caso, en los textos resuntivos la 'interpretación' del texto de otros, ya sea en términos del registro de información básica, su reelaboración, expansión o crítica requiere de cierto nivel de control de la 'interpretación' que se deja explicitada por escrito y esto se vincula con el contenido informativo seleccionado y más o menos comprendido.

En cuanto al uso del PND en estos textos, que es el objeto de referencia de las demás consideraciones, se puede suponer que minimiza, más que otras formas pronominales demostrativas, la incidencia de los conocimientos temáticos previos, al facilitar un recurso de composición más débilmente comprometido con un abordaje comprensivo y elaborativo profundo de los segmentos vinculados y, posiblemente, del propio texto. A este respecto, se podrían plantear hipótesis adicionales sobre las dificultades que un uso poco determinado de este pronombre puede significar para la comprensión posterior del texto elaborado por el propio autor, en un momento posterior, o del elaborado por otro.

c. Como los supuestos contextuales que manejamos integran el tipo de consigna y la tarea que se propone a los estudiantes cabe hacer mención a algunas apreciaciones sobre los resultados obtenidos. La tarea no llevaba nota a promediar durante el desarrollo del curso, esto funciona, seguramente, como una fuente de atenuación de mayores consecuencias sobre la calificación. Presumimos que esto pudo favorecer determinadas condiciones de producción del texto que, por ejemplo, pueden significar un nivel de desempeño acotado por la finalidad funcional de completar la tarea antes que por elaborarla concienzudamente. La escasa extensión de algunos textos podría estar en relación con esto. El pronombre neutro acompañaría esta tendencia, convirtiéndose en un recurso facilitativo de enlaces menos razonados. Poniendo en contraste los textos de uso personal con estos u otros textos semejantes, podría acotarse que esto no implicaría que el resultado de la producción y del uso específico del PDN resultara muy diferente. Sin embargo, puede llamar la atención al contexto de prácticas más amplio en el que se inserta la escritura en el campo de la comunidad de referencia, en este caso, la que nuclea la enseñanza y prácticas académicas y profesionales de la Psicología.

Todo esto sugiere la necesidad de pensar estrategias de trabajo en el contexto de enseñanza que puedan tomar en cuenta resultados semejantes y su significación en 
los procesos de aprendizaje y abordaje de textos, tanto a nivel de su producción como de su lectura y comprensión. El uso de la unidad 'esto' puede interpretarse como un síntoma de diversos aspectos conectados al relacionamiento de los estudiantes con los textos que leen y/o producen y a las condiciones contextuales en que lo hacen. El PDN mostró un ejemplo de nexo anafórico que cobra distintos valores en el texto, y cuya significación es probablemente muy opaca para los estudiantes.

Los investigadores que han atendido a las prácticas y géneros académicos (Bazerman, 1988; Lea \& Street, 1998; Jones, Turner \& Street, 1999; Swales, 2005) podrían aportar ideas relevantes para complementar el análisis de algunas de las implicancias no exentas de debate, teniendo en cuenta, además, que se trata de estudiantes universitarios. A cierta distancia de los debates entre distintas posiciones, podría hacerse notorio, por ejemplo, la importancia de favorecer una reflexión sobre las relaciones entre el texto producido y el TF en sus distintos niveles, abarcando la composición y organización del contenido significativo, la dimensión textuallingüística y comunicativa y el filtro posicional y evaluativo del escritor ante su texto y los de otros, no solo a nivel de la producción, sino a nivel de las distintas interacciones y finalidades que con ellos puedan realizar.

\section{Comentarios finales}

En este trabajo nos hemos ocupado de presentar algunas cuestiones relativas al uso del PDN en la producción escrita de estudiantes universitarios a través de un corpus. Nos orientamos por creer que el uso del PDN puede resultar más preferencial que el de otras fórmulas pronominales demostrativas debido a las posibilidades que facilita. Las perspectivas recientes que abordan los géneros textuales y discursivos tienden a desatender el estudio de unidades textuales al otorgar relevancia al complejo más amplio de producción, en este estudio, se partió de considerar que la concentración en el demostrativo 'esto', tomado en sus diferentes funciones, constituía un pretexto para aproximarse a una complejidad de dimensiones que se conjugan en su uso. Ciertas regularidades que se producen en el manejo contextuado textual y situado de una determinada unidad pueden servir a una comprensión más general de las características del funcionamiento de los textos y de los procesos de lectura y escritura, tanto en miembros habilidosos como en los que se hallan en procesos de formación. De ahí, sus derivaciones educativas, no restrictas a un enfoque formalista y normativo. El PDN, pese a poder considerarse un caso altamente marginal dentro de los problemas asociados al abordaje de textos, tanto en su producción como en su comprensión, permitió aislar y considerar con mayor detalle algunas cuestiones relativas a la lectura y a la escritura que supone reescritura y reformulación de otros textos.

El estudio directo del texto producido en situaciones reales de desempeño de escritores nóveles nos parece de interés para destacar aspectos que desafían y promueven la captación de relaciones que pueden tardar bastante en aparecer en 
el marco de los modelos o perspectivas generales más estabilizadas. Existe mucha investigación de usos característicos en los géneros académicos y/o profesionales que no han prestado atención particular al contexto semántico-pragmático y a las operaciones y estrategias dependientes del género, así como a las tensiones entre principios que subyacen a la composición en interacción con la tarea y las condiciones contextuales. Sería interesante a este respecto complementar este estudio con el contraste con otros textos y tipos de enlaces cohesivos. 


\section{REFERECIAS BIBLIOGRÁFICAS}

Bazerman, C. (1988). Shaping written knowledge. The genre and activity of the experimental article in science. Madison, Wisconsin: University of Wisconsin Press.

Bakthin, M. (1985). La estética de la creación verbal. México, D.F.: Siglo XXI.

Bereiter, C. \& Scardamalia, M. (1987). The psychology of written composition. Hillsdale, NJ: Lawrence Erlbaum.

Bernié, J. P. (1994). Raisonner pour résumer-Une approche systémique des textes. Berne: Peter Lang.

Bernié, J. P. (2001). L'activité de synthèse de documents: Une « compétence »? Quel enjeu? Quel sens donner alors à la notion de « compétence »? En L. Collès, J. L. Dufays, G. Fabry \& C. Maeder (Eds.), Didactique des langues romanes - Le développement de compétences chez l'apprenant (pp. 92-99). Bruxelles: De Boeck \& Duculot.

Bernstein, B. (1971). Class, codes and control: Theoretical studies towards a Sociology of Language. Londres: Routledge \& Kegan Paul.

Bronckart, J. P. (2003). Gêneros textuais, tipos de discursos, e operaçoes psicolingüísticas. Revista de Estudos da Linguagem, 11, 49-69.

Bronckart, J. P. (2004). Les genres de textes et leur contribution au devéloppement psychologique. Langages. Les genres de la parole, 153, 98-108.

Bronckart, J. P. (2006). Atividade de linguagem, discurso e desenvolvimento bumano. Campinas: Mercado de Letras.

Cornish, F. (1995). Référence anaphorique, référence déictique, et contexte prédicatif et énonciatif. Sémiotiques, 8, 31-55.

Cornish, F. (2003). The roles of (written) text and anaphor type distribution in the construction of discourse. Text, 23(1), 1-26.

de Beaugrande, R. \& Dressler, W. (2005). Introducción a la lingüistica del texto. Barcelona: Ariel.

Diessel, H. (1999). Demonstratives. Form, function and grammaticalization. Amsterdam: John Benjamins.

Dolz, J. \& Schneuwly, B. (1997). Les genres scolaires -Des pratiques langagières aux objets d'enseignement. Repères, 15, 27-40.

Ehrlich, M. F. (1996). Metacognitive monitoring in the processing of anaphoric devices in skilled and less skilled comprehenders. En C. Cornoldi \& J. Oakhill (Eds.), Reading comprehension difficulties: Processes and remediation (pp. 221-249). Malwah, NJ: Lawrence Erlbaum.

Eguren, L. J. (1999). Pronombres y adverbios demostrativos. Las relaciones deícticas. En I. Bosque \& V. Demonte (Eds.), Gramática Descriptiva de la Lengua Española. Vol 1. Sintaxis básica de las clases de palabras (pp. 929-972). Madrid: Espasa Calpe.

Finn, S. (1995). Measuring effective writing. Cloze procedure and anaphoric "This". Written Communication, 12(2), 240-266. 
Flower, L., Stein, V., Ackerman, J., Kantz, M. J., McCormick, K. \& Peck, W. C. (1990). Reading-to-Write: Exploring a cognitive and social process. Nueva York: Oxford University Press.

Fuchs, C. (1994). Paraphrase et énonciation. Paris: Ophryz.

Geisler, Ch., Kaufer, D. S. \& Steinberg, E. R. (1985). The unattended anaphoric "This". When should writers use it? Written Communication, 2(2), 129-155.

Givón, T. (2001). Syntax: An introduction. Volume 1. Amsterdam: John Benjamins.

Gülich, E. \& Kotschi, T. (1983). Les marqueurs de reformulation paraphrastique. Cabiers de Linguistique Francaise, 5, 305-351.

Gülich, E. \& Kotschi, T. (1995). Discourse production in oral communication. En U. M. Quasthoff (Ed.), Aspects of oral communication (pp. 30-66). Berlin/New York: de Gruyter.

Halliday, M. A. K. \& Hasan, R. (1976). Cohesion in English. Londres: Longman.

Higgins, L., Flower, L. \& Petraglia, J. (1992). Planning text together: The role of critical reflection in student collaboration. Written Communication, 9(1), 48-84.

Jones, C., Turner, J. \& Street, B. (Eds.) (1999). Students writing in the University: Cultural and epistemological issues. Amsterdam: John Benjamins.

Karmiloff-Smith, A. (1981). The grammatical marking of thematic structure in the development of language production. En W. Deutsch (Ed.), The Child's Construction of Language (pp. 121-147). Nueva York: Academic Press.

Lea, M. \& Street, B. (1998). Student writing in higher education: An academic literacies approach. Studies in Higher Education, 11(3), 182-199.

Lyons, J. (1977). Semantics. Cambridge: Cambridge University Press.

Moskovit, L. (1983). When is broad reference clear? College Composition and Communication, 34(4), 454-469.

O’Brien, E. J., Duffy, S. A. \& Myers, J. L. (1986). Anaphoric inference during reading. Journal of Experimental Psychology: Learning, Memory, and Cognition, 12(3), 346-352.

Poesio, M., Sturt, P., Arstein, R. \& Filik, R. (2006). Underspecification and anaphora: Theoretical issues and preliminary evidence. Discourse Processes, 42(2), 157-175.

Real Academia Española (RAE) (2010). Manual de la nueva gramática de la lengua española. Buenos Aires: Planeta.

Schneuwly, B. (1994). Genres et types de discours: Considérations psychologiques et ontogénétiques. En Y. Reuter (Ed.), Les interactions lecture-écriture (pp. 155-173). Berne: Peter Lang.

Steinberg, E., Kaufer, D. \& Geisler, C. (1984). Response to Leonard Moskovit, When is broad reference clear? College Composition and Communication, 35(4), 478-486.

Stirling, L. (2001). The multifunctionality of anaphoric expressions: A typological perspective. Australian Journal of Linguistics, 21(1), 7-23.

Swales, J. (2005). Attended and unattended "this" in academic writing: A long and unfinished story. ESP Malaysia, 11, 1-15. 
Torres, C. (2004). Itinerarios en la alfabetización académica. Aspectos de la lectura y la escritura en los estudiantes universitarios. En Políticas lingüisticas [CD-ROM]. Instituto de Lingüística, Facultad de Filosofía y Letras (FFyL), Universidad de Buenos Aires, Buenos Aires.

Torres, C. (2009a). Aspectos evolutivos de la reformulación y la función epistémica en producciones escritas intermedias como géneros discursivos. Ponencia presentada en el V Simposio Internacional de Estudios de Géneros Textuales (SIGET), Universidad de Caxias do Sul, Caxias, Brasil.

Torres, C. (2009b). Los esquemas de texto. Aspectos psicolingüísticos, composicionales y textuales a partir de una tarea realizada por estudiantes universitarios. Signo y Seña, 21, 199-217.

van Dijk, T. (1980). Texto y contexto. Madrid: Cátedra.

van Dijk, T. (1983). La ciencia del texto. Barcelona/Buenos Aires: Paidós.

Vigotsky, L. S. (1993). Obras selectas. Tomo II. Madrid: Visor.

Wertsch, J. (1993). Voces de la Mente. Un enfoque sociocultural para el estudio de la Acción Mediada. Madrid: Visor.

Wertsch, J. (1998). Mind as action. Oxford: Oxford University Press.

Wexler, K. \& Chien, Y. (1985). The development of lexical anaphors and pronouns. Papers and Reports on Child Language Development, 24, 138-49.

Yuill, N. \& Oakhill, J. (1988). Understanding of anaphoric relations in skilled and less skilled comprehenders. British Journal of Psychology, 79(2), 173-186.

\section{NOTAS}

1 En el caso del tipo de texto analizado esta diferenciación, no tratada así por otros autores, nos parece relevante debido a la facilidad con que los estudiantes recurren a la copia estricta del texto de la fuente.

2 Podría manejarse la hipótesis de la significatividad que podría asumir el hecho de que apareciera más de una ocurrencia en los textos menos extensos, así como la proporción por número de palabras. De nuestros datos no parece desprenderse correlación con el número de ocurrencias por texto. Podría derivarse de un estudio con corpus más extenso y/o en otros tipos de textos otros datos de interés sobre estos valores numéricos.

3 Esta categoría, poco interesante para interpretar los usos del PDN en cualquier contexto, sirve para consignar el enlace entre TF y texto producido muy específicamente en este tipo de textos. 